\title{
Ellagic acid protects from rotenone-induced dopaminergic neuronal damage via activation of Nrf2 signaling in astroglia
}

\section{Yi-zheng Wei}

Zunyi Medical University

\section{Guo-fu Zhu}

Zunyi Medical University

Chang-qing Zheng

Zunyi Medical University

Jing-jie Li

Zunyi Medical University

Shuo Sheng

Zunyi Medical University

Dai-di Li

Zunyi Medical University

\section{Guo-qing Wang}

Zunyi Medical University

Feng Zhang ( $\sim$ zhangfengzmc@163.com )

Zunyi Medical University

\section{Research}

Keywords: Parkinson's disease, Oxidative stress, Nrf2, Ellagic acid, Neuroprotection

Posted Date: January 8th, 2020

DOI: https://doi.org/10.21203/rs.2.20298/v1

License: (1) (1) This work is licensed under a Creative Commons Attribution 4.0 International License. Read Full License 


\section{Abstract}

\section{Background}

Parkinson's disease (PD) is the second most prevalent central nervous system (CNS) degenerative disease. Oxidative stress is one of key contributors to the degeneration of dopamine (DA) neurons in the pathogenesis of PD. Nuclear factor erythroid-2-related factor 2 (Nrf2) is considered to be a 'master regulator' of many genes involved in anti-oxidant stress to attenuate cell death. Therefore, activation of Nrf2 signaling provides an effective avenue to treat PD. Ellagic acid (EA), a natural polyphenolic contained in fruits and nuts, possesses amounts of pharmacological activities, such as anti-oxidant stress and anti-inflammation. Recent studies have confirmed EA could be used as a neuroprotective agent in neurodegenerative diseases. However, the underlying mechanisms have not yet been entirely elucidated.

\section{Methods}

In this study, mice subcutaneous injection of rotenone (ROT)-induced DA neuronal damage was performed to investigate EA-mediated neuroprotection. In addition, adult Nrf2 knockout mice and different cell cultures including MN9D-enciched, MN9D-BV-2 and MN9D-C6 cell co-cultures were applied to explore the underlying mechanisms.

\section{Results}

The present study demonstrated EA protected DA neurons against ROT-induced neurotoxicity. Furtherly, activation of astroglial Nrf2 signaling pathway participated in EA-mediated neuroprotection as evidenced by the following observations. First, EA activated Nrf2 signaling pathway in ROT-induced DA neuronal injury. Second, EA generated neuroprotection with the presence of astroglia and silence of Nrf2 in astroglia abolished EA-conferred DA neuroprotection. Third, EA failed to produce DA neuroprotection in Nrf2 knockout mice.

\section{Conclusion}

This study identified EA protected against DA neuronal loss via an Nrf2-dependent manner in astroglia.

\section{Background}

To date, the pathogenesis of Parkinson's disease (PD) is intangible [1]. It has been verified that the increased and prolonged production of reactive oxygen species (ROS) plays critical roles in progress of PD [2]. ROS compromises the balances between oxidant and anti-oxidant systems [3]. The anti-oxidant defense system accounted for one of the underlying mechanisms that prevent oxidative stress-induced cell damage. 
Nuclear factor E2-related factor 2 (Nrf2) regulates the physiological and pathophysiological processes of various diseases [4]. Importantly, Nrf2 increases the gene expressions of endogenous anti-oxidative enzymes, such as phase Il detoxifying enzymes, quinone oxidoreductase 1 (NQO1) and $\mathrm{NAD}(\mathrm{P}) \mathrm{H}$, heme oxygenase-1 (HO-1) [5]. A number of studies confirmed that Nrf2 is located and maintained in the cytoplasm in physiological conditions via a Kelch-like ECH-associated protein 1 (Keap1)-dependent ubiquitination-proteasomal degradation [6]. Upon stimulated by oxidants or electrophiles, Nrf2 compromises Keap1/Nrf2 interactions and then Nrf2 dissociates from Keap1 complex and finally enters the nucleus. This is followed by binding to the anti-oxidant response elements (ARE), a consensus gene sequence that encodes anti-oxidant enzymes [7]. Amounts of studies identified that Nrf2 signaling participates in PD pathogenesis [8]. Activation of Nrf2 evoked dopamine (DA) neuroprotection and downregulation of Nrf2 rendered DA neurons susceptible to oxidative stress damage [9]. Insufficient Nrf2 activation was highly involved in the progress of PD [10]. Thus, potent Nrf2 signaling activation is thought to be a potential promising strategy for PD treatment.

Ellagic acid (2, 3, 7, 8-tetrahydroxybenzopyrano [5, 4, 3-cde] benzopyran-5-10- dione, EA) is located abundantly in plant extracts [11]. EA presents a variety of pharmacological properties, such as antiinflammatory, cardioprotective and anti-oxidant effects [12]. Recently, several lines of evidence verified that EA conferred neuroprotection against aging, nerve dysfunction and neurodegeneration. In sporadic Alzheimer's disease animal model, EA generated neuroprotection and cognitive enhancement [13]. In addition, EA protected against sodium arsenate-induced neurotoxicity in rats [14]. Moreover, EA was indicated to produce neuroprotection against ischemic stroke [15]. However, the underlying mechanisms remain unclear.

In the present study, subcutaneous injection of rotenone (ROT)-elicited PD mouse model was employed to investigate EA-exerted DA neuroprotection. Furtherly, adult Nrf2 knockout mice and various cell cultures were applied to illuminate the role of Nrf2 signaling in EA-mediated DA neuroprotection.

\section{Materials And Methods}

\section{Reagents}

Ellagic acid (purity $>95 \%$ ) and rotenone were obtained from Sigma Chemical (St. Louis, MO, USA). Antityrosine hydroxylase (TH, Catalog No. Ab113), hemeoxygenase-1 (HO-1, Catalog No. Ab68477), NADPH quinone oxidoreductase 1 (NQ01, Catalog No. ER1802-86), Nrf2 (Catalog No. Ab31163), PCNA (Catalog No. 10205-2-AP), HSP90 (Catalog No. GB11284-1) and $\beta$-actin antibodies were bought from Abcam (Cambridge, MA, USA). The small interfering RNA (siRNA) against Nrf2 was purchased from Thermo Fisher Scientific (Waltham, MA, USA). SYBR green supermix (PCR) was obtained from Bio-Rad Laboratories (CA, USA). RNAiso plus was purchased from Takara Biotech Co., Ltd. (Dalian, China).

\section{Animals and Treatment}


Wild type (WT) C57BL/6J male mice and homozygous Nrf2 knockout ( $\mathrm{Nrf}^{-/-}$) male mice (22-28 g, 1012 weeks) were purchased from the Nanjing University Biomedical Research Institute (Nanjing, China; Permit number: SCXK2015-0001). All animals were kept in accordance with Chinese animal health and welfare norms. This study was approved by the Animal Care and Use Committee of Zunyi Medical University (Zunyi, China). WT and Nrf2 knockout mice were randomly divided into Control, EA (100 mg/kg), ROT (1 mg/kg), ROT + EA (20 mg/kg) and ROT + EA (100 mg/kg) groups. Mice were given subcutaneous injection of ROT ( $1 \mathrm{mg} / \mathrm{kg}) 6$ times a week for consecutive 5 weeks followed by daily intragastric administration of EA for consecutive another five weeks.

\section{Rotarod Test}

The rotarod test, in which animals must balance on the rod, was widely used to evaluate motor deficit in neurodegenerative disease models. Before the trial, all mice received rotarod training until they stayed on the stick at least for the cut-off time. In the test, the started speed was $10 \mathrm{rpm}$ and every $30 \mathrm{~s}$ increased $5 \mathrm{rpm}$ until mice slid off the steps. Every mouse was tested 2 times a day and the average duration of stay on the rod was recorded.

\section{Open Field Test}

Open field test was one of the most popular ethological tests to detect the anxiety-like behavior in animals. In the present study, mice were placed on the open field and each animal was located in a separate area and mice behavioral parameters were recorded during 5 min [16]. Before each round of testing, the device should be cleaned with a $75 \%$ alcohol solution to eliminate odor interference. After the end of the experiment, the total distance of mice movement was calculated.

\section{Immunohistochemistry Staining and Cell Counting}

Mice brains were cut into 7 microns cross sections on a paraffin slicer and attached to a glass slide. The brain slices were then dried and dewaxed. Fixed brain slices were separately treated with $3 \%$ hydrogen peroxide and $0.1 \mathrm{M}$ citrate buffer, and blocked with goat serum. Next, it was cultured overnight with an anti-TH antibody at $4{ }^{\circ} \mathrm{C}$. Subsequently, brain sections were incubated with the secondary antibody working solution for $20 \mathrm{~min}$ followed by sections incubated with biotin for $15 \mathrm{~min}$ and developed with DAB developer. For morphological analysis, Olympus microscope (Tokyo, Japan) was applied to image $\mathrm{TH}$-positive neurons in SN. Finally, TH-positive neurons were counted through counting the number of THpositive neuronal cell bodies blindly by two investigators and the results were averaged.

\section{Western Blotting}

Total protein was extracted from mice midbrain by RIPA lysis solution. Nuclear and cytosolic fractions were extracted using a nuclear-cell solute extraction kit (Solabio, Beijing, China). The protein concentrations were quantified by a BCA kit. Equal amounts of protein $(10-30 \mu \mathrm{g} / \mathrm{lane})$ were separated on a $10 \%$ Bis-Tris Nu-PAGE gel. Then, proteins were transferred to a polyvinylidene fluoride (PVDF) membrane, blocked with $8 \%$ skim milk for $2.5 \mathrm{~h}$, and placed in the primary antibody at $4{ }^{\circ} \mathrm{C}$ overnight. The primary antibodies were: TH (1:2000), Nrf2 (1:1000), HO-1 (1:10000), NQO1 (1:1000), PCNA (1:5000) and 
$\beta$-actin (1: 3000). Next, membranes were incubated with anti-rabbit/mouse IgG secondary antibody at 1:2000 for $1 \mathrm{~h}$ and detected with ECL substrate. The results were statistically analyzed by quantitative analysis (Bio-Rad, Hercules, CA, USA) software.

\section{Real-Time RT-PCR Assay}

Total RNA in the mice midbrain and cells were separately extracted by RNAiso plus reagent and purified with RNeasy Kits. The SYBR Green PCR Master Mix was used for real-time PCR analysis. The primers used were designed by Sangon Biotech (Shanghai, China). The relative differences of the target genes were first normalized to $\beta$-actin, and then calculated and expressed as a relative reduction or increase, setting the Control group at $100 \%$.

\section{Cell Culture and Treatment}

BV-2 microglia cell lines, C6 astroglia cell lines and MN9D DA neuron cell lines were obtained from the Chinese Academy of Sciences Cell Bank (Shanghai). All cultures were maintained in DMEM/F12 media supplemented with $10 \%$ fetal bovine serum and $1 \%$ antibiotics. Three kinds of cell lines were cultured at $37{ }^{\circ} \mathrm{C}$ in a humidified atmosphere of $5 \% \mathrm{CO}_{2}$ and $95 \%$ air. Cells were seeded at $1 \times 10^{6} /$ well in poly-Dlysinecoated 24-well plates, respectively. Cells were treated with different concentrations of EA for 30 min followed by ROT $(0.1 \mu \mathrm{M})$ treatment for an additional $24 \mathrm{~h}$. Then, cells and culture medium were collected for later detection.

\section{Nrf2-siRNA Transfection}

C6 astroglia cell lines were seeds on 24-well plates for $24 \mathrm{~h}$ and then transfected with Nrf2-siRNA $(50 \mathrm{nmol} / \mathrm{L})$ or control-siRNA $(50 \mathrm{nmol} / \mathrm{L})$ for $6 \mathrm{~h}$ using siRNA transfection reagent (Thermo Fisher Scientific, Massachusetts, USA) using the manufacturer's protocol. The knockdown efficiency was evaluated by western blotting and real-time RT-PCR. After $6 \mathrm{~h}$ of transfection, the transfection solution was removed and cells were treated with EA for $24 \mathrm{~h}$ followed by ROT administration for $24 \mathrm{~h}$. Afterwards, C6 cells were collected to detect the corresponding indicators.

\section{Reconstituted Neuron-Microglia and Neuron-Astroroglia Co- Cultures Using Transwells}

BV-2 cells or C6 cells were cultured in the upper chamber of transwell inserts for $24 \mathrm{~h}$. The lower chambers contained MN9D cells were also cultured for $24 \mathrm{~h}$. Then, BV-2 or C6 cells in transwells were transferred into MN9D cells and the reconstituted neuron-microglia (MN9D-BV-2) and neuron-astroglia (MN9D-C6) co-cultures were established. These co-cultures were treated with EA for 30 min followed by ROT application for $24 \mathrm{~h}$. For MN9D-C6 co-cultures, the C6 cells in transwells were processed by Nrf2siRNA for $6 \mathrm{~h}$. Then, the MN9D cells in lower chambers were cultured for $24 \mathrm{~h}$. Next, C6 cells were rinsed with fresh medium and transferred into MN9D cultures. The reconstituted neuron-astroglia co-cultures conditionally silencing astroglia Nrf2 were established. Finally, cultures were treated with EA for 30 min followed by ROT treatment for $24 \mathrm{~h}[17,18]$. DA neuronal damage was assessed by TH-positive neuron staining and TH protein expression. 


\section{Cell Viability Assay}

After EA treatment for $24 \mathrm{~h}$, the upper chambers were moved and the lower chambers were incubated with MTT solution ( $5 \mathrm{mg} / \mathrm{ml}$ ) for $4 \mathrm{~h}$. Dimethyl sulfoxide (DMSO) was added to each well to determine cell viability. Absorbance values were measured at $490 \mathrm{~nm}$.

\section{Immunocytochemical Staining}

DA neurons were recognized with an anti-TH antibody. Cells were fixed with $4 \%$ paraformaldehyde followed by permeabilization using $0.03 \%$ Triton X-100 and closed with goat serum. DA neurons were labeled with an anti-TH (1:300) antibody at $4{ }^{\circ} \mathrm{C}$ overnight and then incubated with an anti-rabbit-lgG (1:2000) antibody for $1 \mathrm{~h}$. TH-positive neurons numbers were calculated from four representative areas per well of the 24-well plate. In each condition, three wells were used for cell counting.

\section{Statistical Analysis}

Data analyses were performed as mean \pm SEM. Statistical comparisons were analyzed using the SPSS statistical software by one-way ANOVA. Then, the Bonferroni's post hoc test was performed for all pairwise comparisons among means. $p<0.05$ was considered as statistically significant.

\section{Results}

\section{EA protected against ROT-induced DA neuronal damage}

To explore the neuroprotective effects of EA on PD, we established ROT-induced DA neuronal lesion in mice and determined whether EA could attenuate ROT-induced DA neurotoxicity. First, we examined behavior changes by open field and rotarod tests. As shown in Fig. 1a and b, ROT caused an apparent reduction in total movement distance and the time mice stayed on the rod. EA exhibited a significant improvement in locomotor dysfunction. Furthermore, ROT decreased TH-positive neuronal number and TH protein expression and EA ameliorated ROT-induced DA neuronal damage (Fig. 1c and d).

EA activated Nrf2 signaling pathway in vivo

To determine whether EA could activate Nrf2 signaling pathway, the expressions of Nrf2, HO-1, and NQO1 were detected by real-time RT-PCR and western blot assay. As shown in Fig. 2a, the mRNA expressions of Nrf2, HO-1 and NQO1 were increased in the ROT and EA groups, in which EA induced higher mRNA expressions of these genes than ROT. To further determine the effects of EA on Nrf2 distribution, the cytosolic components and nuclear fractions were collected, respectively. The cytosol marker HSP90 was used to verify the purity of nuclear protein and the nuclear fraction was not contaminated by cytosol proteins as confirmed by the absence of HSP90. As shown in Fig. 2b, compared with control group, ROT activated Nrf2 and induced Nrf2 to transfer from cytosol to nucleus. Compared with ROT group, the increase in translocation of Nrf2 from cytosol to nucleus was more pronounced in EA group. Meanwhile, 
the higher protein expressions of HO-1 and NQO1 in ROT + EA group were indicated than those in ROT group (Fig. 2c).

\section{EA targeted astroglia to protect from ROT-induced DA neuronal damage}

To further explore which cell type EA targeted to produce DA neuroprotection, three cell culture systems including MN9D-enriched, MN9D-BV2 and MN9D-C6 co-cultures were performed using transwells. Cultures were treated with EA for 30 min followed by ROT treatment for $24 \mathrm{~h}$. As shown in Fig. 3a, EAmediated neuroprotection was indicated in MN9D-C6 co-culture system but not in either MN9D-enriched or MN9D-BV2 co-cultures. Similarly, from TH-positive neuronal immunofluorescence staining and TH protein detection assays shown in Fig. 3b and c, in MN9D-C6 co-culture system, EA attenuated ROTinduced decrease of TH-positive neuronal number and TH protein expression. However, in MN9D-enriched cultures and MN9D-BV2 co-cultures, this neuroprotection disappeared. These phenomenon demonstrated astroglia participated in EA-elicited neuroprotection.

EA activated Nrf2 signaling pathway in vitro

To confirm the activation of Nrf2 signaling induced by EA treatment in which cell type, the expressions of Nrf2 signaling in MN9D, BV2 and C6 cells were detected. As shown in Fig. 4a, Nrf2 protein expression mainly concentrated in $\mathrm{C} 6$ cells. To further determine whether EA activated Nrf2 signaling in astroglia, the mRNA and protein expressions of Nrf2 signaling in $\mathrm{C} 6$ cells were examined. As shown in Fig. 4b, Nrf2, HO-1 and NQ01 mRNA levels were up-regulated in ROT and ROT + EA treatment groups, whereas EA enhanced these genes mRNA expressions compared with ROT administration. In addition, western blot assay showed the translocation of Nrf2 from cytoplasm to the nucleus was more obvious after EA treatment that that in ROT group (Fig. 4c). Also, EA induced the higher protein expressions of HO-1 and NQ01 than ROT (Fig. 4d).

\section{EA protected DA neurons by the activation of astroglial Nrf2}

To explore the role of Nrf2 in EA-mediated DA neuroprotection, Nrf2 siRNA in vitro and Nrf2 knockout mice were performed. As shown in Fig. 5a, Nrf2 gene and protein levels were down-regulated in C6 cells transfected with Nrf2-siRNA with the silence ratio of $70 \%$. Furtherly, in TH-positive neuronal counting and TH protein expression assays, EA-generated DA neuroprotection from ROT-induced neurotoxicity was neutralized by Nrf2-siRNA application (Fig. 5b and c).

Next, Nrf2 knockout mice were employed to confirm EA evoked neuroprotection via an Nrf2-dependent manner in vivo. First, the knockout efficiency was verified by Nrf2 protein level detection (Fig. 6a). Then, there was no significant difference of the genes and proteins expressions of HO-1 and NQO1 in the midbrain of Nrf2 knockout mice after EA treatment (Fig. 6b and c). In addition, EA failed to improve the time mice stayed on rod and locomotor distance in Nrf2 knockout mice (Fig. 6d and e). Meanwhile, TH- 
positive neuronal counting and TH protein expression detection showed EA-mediated DA neuroprotection was abolished in Nrf2 knockout mice (Fig. $6 \mathrm{f}$ and g). Collectively, these results demonstrated EA protected DA neurons through the activation of astroglial Nrf2 signaling.

\section{Discussion}

The present study aimed at investigating the neuroprotective actions of EA on ROT-induced DA neuronal damage and the underlying mechanisms as well. Results demonstrated that EA conferred neuroprotection against ROT-induced DA neurotoxicity. Activation of astroglial Nrf2 signaling was involved in EA-mediated DA neuroprotection, as evidenced by the following observations. First, EA activated Nrf2 signaling pathway in ROT-induced DA neuronal damage. Second, EA generated neuroprotection with the presence of astroglia and silence of Nrf2 in astroglia abolished EA-mediated neuroprotection. Third, EA failed to produce DA neuroprotection in Nrf2 knockout mice. Together, these results suggested EA-generated DA neuroprotection might be attributable to the activation of Nrf2 signaling in astroglia.

Oxidative stress has been considered as the central pathological event in the development of PD $[19,20]$. For survival and development of neurons, the maintenance of redox homeostasis in the CNS was indispensable [21]. Nrf2 was an oxidative defense transcription factor which mitigated the toxic neuronal effects of parkinsonian agents, such as ROT, 1-methyl-4-phenyl-1,2,3,6-tetrahydropyridine (MPTP) and 6hydroxydopamine (6-OHDA) in vivo and in vitro [22]. Here, this study found that EA protected DA neurons, activated Nrf2 signaling pathway and initiated the Nrf2 stressor mechanism for its transfer from cytosol to the nucleus (Figs. 1 and 2). Further study indicated that EA-elicited neuroprotection were abolished in Nrf2 knockout mice (Fig. 6). These results suggested that EA-enhanced DA neuronal survival was dependent on Nrf2 activation.

Encouraged by the activation of Nrf2 signaling by EA, we further investigated Nrf2 signaling in which cell type participated in EA-mediated neuroprotection. It is interesting to note that EA targeted astroglia to produce neuroprotection since this protection was just indicated in the presence of astroglia (Fig. 3). Astroglia is the most abundant cell type in the brain [23]. They interacted with neurons in various aspects of signaling transmission, immune and oxidative defense, ion and water homeostasis, metabolite supply and neuroprotection [24]. In neurological disorders, astroglia themselves formed a defense barrier and promoted neuroprotection by producing anti-oxidants against inflammation, especially for excessive extracellular release of the excitatory neurotransmitter glutamate [25]. Stimulation of sulforaphane also prolonged Nrf2-mediated gene expression in astrocytes and prevented neuronal damage caused by superoxide [26]. In addition, besides anti-oxidants, astrocytes could release neurotrophic factors, such as BDNF and GDNF-mediated activation of the Nrf2 pathway in astrocytes, which increased the survival of DA neurons [27]. Recent research indicated that Nrf2-ARE pathway was preferentially activated in astrocytes, HO-1 and NQ01were strongly expressed in astrocytes, sometime microglia, with more infrequent expression in neurons $[28,29]$. Nevertheless, Pharmacological activation and overexpression of Nrf2 in astrocytes also clearly demonstrated its therapeutic potential for PD. In the mouse model of a- 
synuclein mutation, the specific overexpression of astrocyte Nrf2 was regulated, which improved the movement and non-motor dysfunction of the whole CNS, and delayed the process of a-synuclein aggregation [30]. Also, Nrf2 overexpressed in astrocytes kept mice from mutated a-synuclein to protect DA neurons [31]. Additionally, transplantation of astrocytes overexpressing Nrf2 into mouse striatum protected from DA neuronal loss [32]. Therefore, this was an important strategy for the support of neuroprotective functions of astrocytes to enhance neuronal survival and improve PD symptoms.

A large amount of evidence suggested Nrf2 activators could protect neurons and decrease the accumulation of aberrant proteins in vitro and in vivo in different neurodegenerative mouse models. In clinic, melatonin was found to activate Nrf2 to rescue hippocampal bioenergetics and improve cognitive function [33]. At present, the clinical trials on Nrf2 activators for the treatment of neurodegenerative diseases were increasing [34]. For example, pinostrobin could release HO-1 through Nrf2 activation to alleviate MPTP-induced neuronal loss in SN, and relieved zebra fish behavioral deficit disorder, and provided potential neuroprotective effects in vivo [25]. Moreover, Glaucocalyxin B was discerned to inhibit TLR/NF-KB pathway activation and induce Nrf2/HO-1 activation and further produce neuroprotection [35]. Also, dimethyl fumarate enhanced Nrf2 activation, thereby playing an important role in the defense of MPTP-induced loss of DA neurons in the SN [36]. In short, new Nrf2-based treatments might be considered as a viable strategy for future potential. Thus, this study found EA exhibited a promising therapeutic efficacy for PD via activating Nrf2 signaling pathway. These findings suggested that modulation of Nrf2 signaling by potential candidates, such as EA, might be beneficial for PD treatment. Despite this optimistic perspective for future administration of EA potential treatment for PD, most of the findings were derived from experimental models and in vitro data and therefore need be rigorously corroborated in clinical trials in future.

\section{Conclusions}

This study demonstrated that EA protected DA neurons from ROT-induced neurotoxicity by activating astroglial Nrf2 signaling. These findings suggested EA might be an exciting option for treating PD.

\section{Abbreviations}
EA Ellagic acid

CNS Central Nervous System

ARE Anti-oxidant response elements

DA Dopamine

ROT Rotenone

HO-1 Heme oxygenase-1 
NQ01 NADPH quinone oxidoreductase 1

Nrf2 Nuclear factor erythroid-2 related factor 2

PCNA Proliferating cell nuclear antigen

PD Parkinson's disease

ROS Reactive oxygen species

SN Substantia nigra

TH Tyrosine hydroxylase

WT Wild type

Nrf2 KO Nrf2 knockout

6-OHDA 6-hydroxydopamine

MPTP 1-methyl-4-phenyl-1,2,3,6-tetrahydropyridine

\section{Declarations}

\section{Availability of data and materials}

The datasets used and/or analyzed during the current study are available from the corresponding author on reasonable request.

\section{Acknowledgements}

We are grateful to the technical support provided by Shanghai Baiqu

Biomedical Technology Co., Ltd.

\section{Funding}

All experiments were supported by National Natural Science Foundation of China (no. 81760658), Highlevel Innovative Talents of Guizhou Province (no. 20164027), the Innovation Research Group Project of Education Department of Guizhou Province (no. 2016038), the foundation for Excellent Young Talents of Zunyi Medical University (no. 201603).

\section{Author contribution}

FZ conceived and designed the experiments. All the authors participated in the experiment performance and data analysis. FZ, GQW and YZW wrote, revised and checked the article. All authors revised and 
approved the final manuscript.

\section{Ethics approval and consent to participate}

All experimental procedures were carried out in accordance with Chinese

Guidelines of Animal Care and Welfare and this study received an approval

from the Animal Care and Use Committee of Zunyi Medical University

(Zunyi, China).

\section{Consent for Publication}

Written informed consent for publication was obtained from all participants.

\section{Competing interests}

The authors declared no conflicts of interests.

\section{Publisher's Note}

Springer Nature remains neutral with regard to jurisdictional claims in published maps and institutional affiliations.

\section{References}

1. Panicker N, Saminathan H, Jin H, Neal M, Harischandra DS, Gordon R, Kanthasamy K, Lawana V, Sarkar S, Luo J, Anantharam V, Kanthasamy AG and Kanthasamy A. Fyn Kinase Regulates Microglial Neuroinflammatory Responses in Cell Culture and Animal Models of Parkinson's Disease. J Neurosci $2015 ; 35: 10058-77$

2. Maiti P, Manna J and Dunbar GL. Current understanding of the molecular mechanisms in Parkinson's disease: Targets for potential treatments. Transl Neurodegener 2017; 6:28

3. Tansey MG, McCoy MK and Frank-Cannon TC. Neuroinflammatory mechanisms in Parkinson's disease: potential environmental triggers, pathways, and targets for early therapeutic intervention. Exp Neurol 2007; 208:1-25

4. AlDakheel A, Kalia LV and Lang AE. Pathogenesis-targeted, disease-modifying therapies in Parkinson disease. Neurotherapeutics 2014; 11:6-23

5. Yan J, Li J, Zhang L, Sun Y, Jiang J, Huang Y, Xu H, Jiang H and Hu R. Nrf2 protects against acute lung injury and inflammation by modulating TLR4 and Akt signaling. Free Radic Biol Med 2018; 121:78-85

6. Ruiz S, Pergola PE, Zager RA and Vaziri ND. Targeting the transcription factor Nrf2 to ameliorate oxidative stress and inflammation in chronic kidney disease. Kidney Int 2013; 83:1029-41 
7. Zhao Y, Song W, Wang Z, Wang Z, Jin X, Xu J, Bai L, Li Y, Cui J and Cai L. Resveratrol attenuates testicular apoptosis in type 1 diabetic mice: Role of Akt-mediated Nrf2 activation and p62-dependent Keap1 degradation. Redox Biol 2018; 14:609-617

8. Dheen ST, Jun Y, Yan Z, Tay SS and Ling EA. Retinoic acid inhibits expression of TNF-alpha and iNOS in activated rat microglia. Glia 2005; 50:21-31

9. Lastres-Becker I, Garcia-Yague AJ, Scannevin RH, Casarejos MJ, Kugler S, Rabano A and Cuadrado A. Repurposing the NRF2 Activator Dimethyl Fumarate as Therapy Against Synucleinopathy in Parkinson's Disease. Antioxid Redox Signal 2016; 25:61-77

10. Vargas MR, Pehar M, Cassina P, Beckman JS and Barbeito L. Increased glutathione biosynthesis by Nrf2 activation in astrocytes prevents p75NTR-dependent motor neuron apoptosis. J Neurochem 2006; 97:687-96

11. Neymotin A, Calingasan NY, Wille E, Naseri N, Petri S, Damiano M, Liby KT, Risingsong R, Sporn M, Beal MF and Kiaei M. Neuroprotective effect of Nrf2/ARE activators, CDDO ethylamide and CDDO trifluoroethylamide, in a mouse model of amyotrophic lateral sclerosis. Free Radic Biol Med 2011; 51:88-96

12. Firdaus F, Zafeer MF, Anis E, Ahmad M and Afzal M. Ellagic acid attenuates arsenic induced neuroinflammation and mitochondrial dysfunction associated apoptosis. Toxicol Rep 2018; 5:411-417

13. Jha AB, Panchal SS and Shah A. Ellagic acid: Insights into its neuroprotective and cognitive enhancement effects in sporadic Alzheimer's disease. Pharmacol Biochem Behav 2018; 175:33-46

14. Goudarzi M, Amiri S, Nesari A, Hosseinzadeh A, Mansouri E and Mehrzadi S .The possible neuroprotective effect of ellagic acid on sodium arsenate-induced neurotoxicity in rats. Life Sci 2018; 198:38-45

15. Liu QS, Deng R, Li S, Li X, Li K, Kebaituli G, Li X and Liu R. Ellagic acid protects against neuron damage in ischemic stroke through regulating the ratio of Bcl-2/Bax expression. Appl Physiol Nutr Metab 2017; 42:855-860

16. Kraeuter AK, Guest PC and Sarnyai Z. The Open Field Test for Measuring Locomotor Activity and Anxiety-Like Behavior. Methods Mol Biol 2019; 1916:99-103

17. Bates AM, Fischer CL, Abhyankar VP, Johnson GK, Guthmiller JM, Progulske-Fox A and Brogden KA. Matrix Metalloproteinase Response of Dendritic Cell, Gingival Epithelial Keratinocyte, and T-Cell Transwell Co-Cultures Treated with Porphyromonas gingivalis Hemagglutinin-B. Int J Mol Sci 2018;19

18. Chen SH, Oyarzabal EA, Sung YF, Chu CH, Wang Q, Chen SL, Lu RB and Hong JS. Microglial regulation of immunological and neuroprotective functions of astroglia. Glia 2015; 63:118-31

19. Surendran $S$ and Rajasankar S. Parkinson's disease: oxidative stress and therapeutic approaches. Neurol Sci 2010; 31:531-40

20. Guo JD, Zhao X, Li Y, Li GR and Liu XL. Damage to dopaminergic neurons by oxidative stress in Parkinson's disease (Review). Int J Mol Med 2018; 41:1817-1825 
21. Xicoy H, Wieringa B and Martens GJ. The SH-SY5Y cell line in Parkinson's disease research: a systematic review. Mol Neurodegener 2017; 12:10

22. Huang B, Liu J, Meng T, Li Y, He D, Ran X, Chen G, Guo W, Kan X, Fu S, Wang W and Liu D. Polydatin Prevents Lipopolysaccharide (LPS)-Induced Parkinson's Disease via Regulation of the AKT/GSK3beta-Nrf2/NF-kappaB Signaling Axis. Front Immunol 2018; 9:2527

23. Gorshkov K, Aguisanda F, Thorne N and Zheng W. Astrocytes as targets for drug discovery. Drug Discov Today 2018; 23:673-680

24. Rivetti di Val Cervo P, Romanov RA, Spigolon G, Masini D, Martin-Montanez E, Toledo EM, La Manno G, Feyder M, Pifl C, Ng YH, Sanchez SP, Linnarsson S, Wernig M, Harkany T, Fisone G and Arenas E. Induction of functional dopamine neurons from human astrocytes in vitro and mouse astrocytes in a Parkinson's disease model. Nat Biotechnol 2017; 35:444-452

25. Liu B, Teschemacher AG and Kasparov S. Astroglia as a cellular target for neuroprotection and treatment of neuro-psychiatric disorders. Glia 2017; 65:1205-1226

26. Bergstrom P, Andersson HC, Gao Y, Karlsson JO, Nodin C, Anderson MF, Nilsson M and Hammarsten O. Repeated transient sulforaphane stimulation in astrocytes leads to prolonged Nrf2-mediated gene expression and protection from superoxide-induced damage. Neuropharmacology 2011; 60:343-53

27. Ishii T, Warabi E and Mann GE. Circadian control of BDNF-mediated Nrf2 activation in astrocytes protects dopaminergic neurons from ferroptosis. Free Radic Biol Med 2019; 133:169-178

28. Joe EH, Choi DJ, An J, Eun JH, Jou I and Park S. Astrocytes, Microglia, and Parkinson's Disease. Exp Neurobiol 2018; 27:77-87

29. de Freitas Silva M, Pruccoli L, Morroni F, Sita G, Seghetti F, Viegas C and Tarozzi A. The Keap1/Nrf2ARE Pathway as a Pharmacological Target for Chalcones. Molecules 2018; 23

30. Gan L, Vargas MR, Johnson DA and Johnson JA. Astrocyte-specific overexpression of Nrf2 delays motor pathology and synuclein aggregation throughout the CNS in the alpha-synuclein mutant (A53T) mouse model. J Neurosci 2012; 32:17775-87

31. Chen PC, Vargas MR, Pani AK, Smeyne RJ, Johnson DA, Kan YW and Johnson JA. Nrf2-mediated neuroprotection in the MPTP mouse model of Parkinson's disease: Critical role for the astrocyte. Proc Natl Acad Sci U S A 2009; 106:2933-8

32. Liddell JR. Are Astrocytes the Predominant Cell Type for Activation of Nrf2 in Aging and Neurodegeneration? Antioxidants (Basel) 2017; 6

33. Chen LY, Renn TY, Liao WC, Mai FD, Ho YJ, Hsiao G, Lee AW and Chang HM. Melatonin successfully rescues hippocampal bioenergetics and improves cognitive function following drug intoxication by promoting Nrf2-ARE signaling activity. J Pineal Res 2017; 63

34. Houghton CA, Fassett RG and Coombes JS. Sulforaphane and Other Nutrigenomic Nrf2 Activators: Can the Clinician's Expectation Be Matched by the Reality? Oxid Med Cell Longev 2016; 2016:7857186

35. Xu W, Zheng D, Liu Y, Li J, Yang L and Shang X. Glaucocalyxin B Alleviates LipopolysaccharideInduced Parkinson's Disease by Inhibiting TLR/NF-kappaB and Activating Nrf2/HO-1 Pathway. Cell 
Physiol Biochem 2017; 44:2091-2104

36. Campolo M, Casili G, Biundo F, Crupi R, Cordaro M, Cuzzocrea S and Esposito E. The Neuroprotective Effect of Dimethyl Fumarate in an MPTP-Mouse Model of Parkinson's Disease: Involvement of Reactive Oxygen Species/Nuclear Factor-kappaB/Nuclear Transcription Factor Related to NF-E2. Antioxid Redox Signal 2017; 27:453-471

\section{Figures}

a

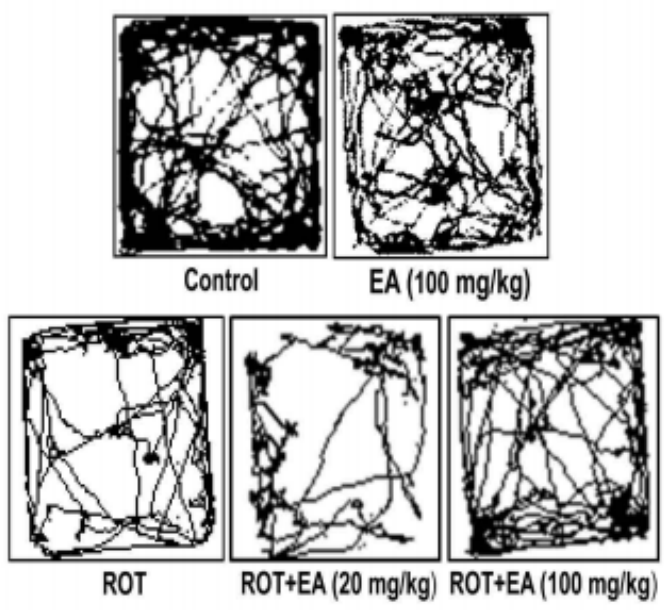

C

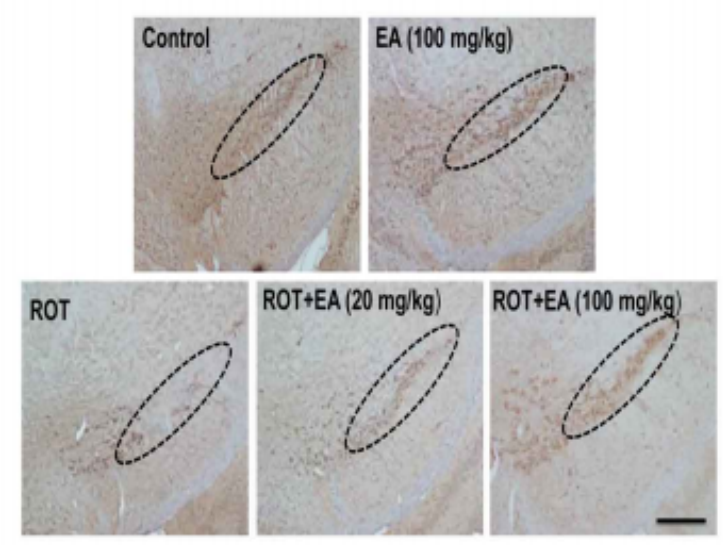

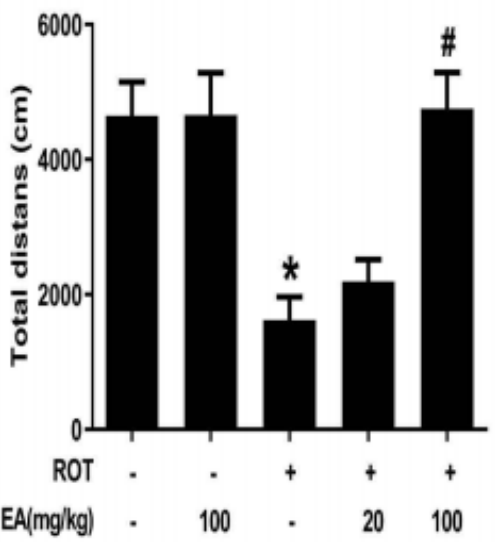

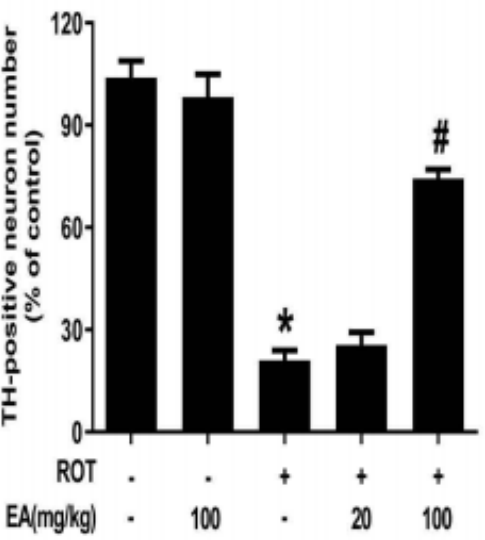

b

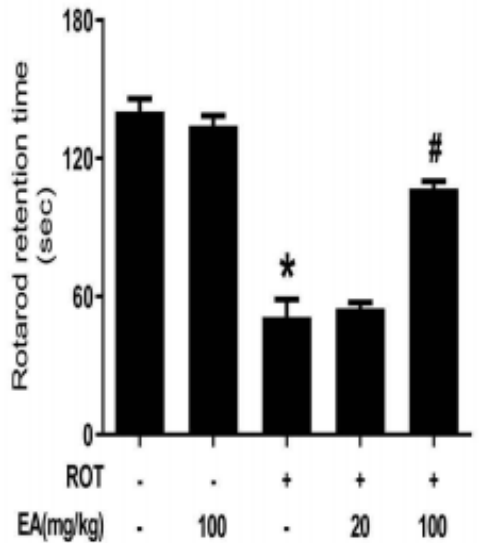

d

$\mathrm{EA}(\mathrm{mg} / \mathrm{kg})$ Marker $\cdot 100 \quad \cdot 20100$

TH
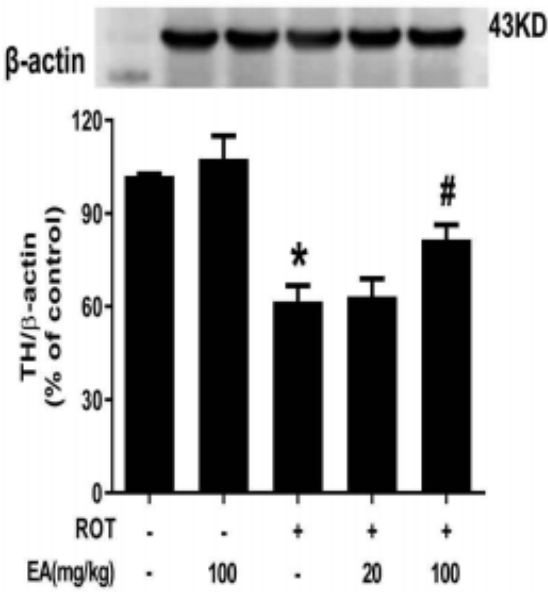

Figure 1

EA ameliorated ROT-induced DA neuronal damage. Male wild type mice received subcutaneous injection of ROT $(1 \mathrm{mg} / \mathrm{kg})$ six times a week for consecutive five weeks. EA were daily given by intragastric administration for consecutive another five weeks. a, b Open field test and rotarod test were performed to evaluate animal behavior changes. c TH-positive neurons number was counted after midbrain sections immunohistochemistry staining. Scale bar $=200 \mu \mathrm{m}$. d TH protein expression was tested by western blot 
assay. Results were represented as mean \pm SEM from 6 mice. ${ }^{*} p<0.05$ compared with the control group, \#p<0.05 compared with ROT-treated group.

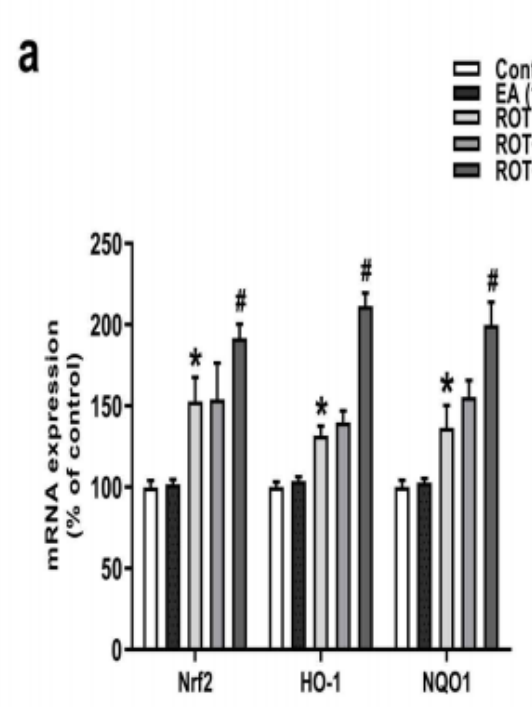

C

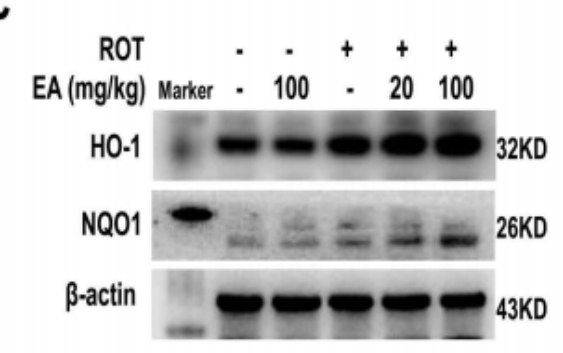

b

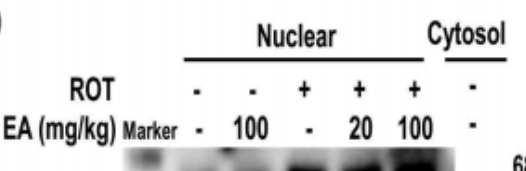

Nrf2 $=700 \mathrm{COKD}$

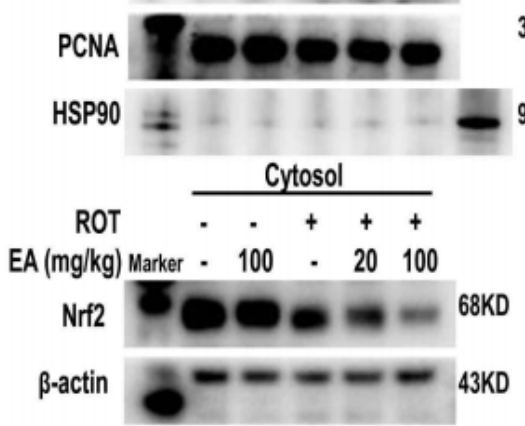

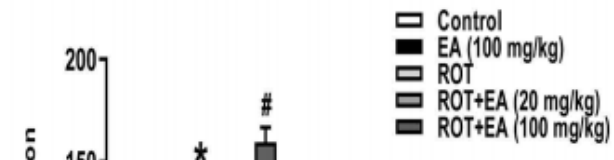

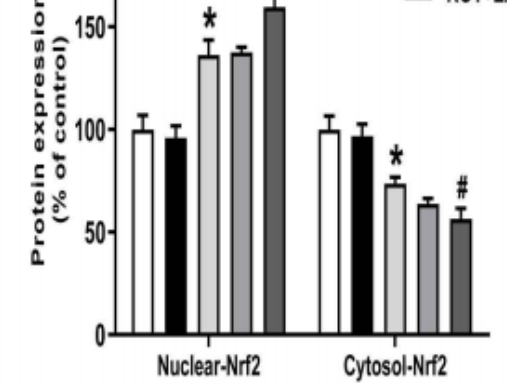

므남ol

EA(100 mglkg)

믈 ROTEA $20 \mathrm{mg} / \mathrm{kg})$

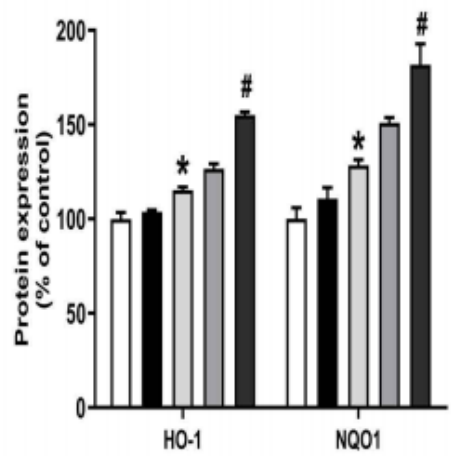

\section{Figure 2}

EA activated Nrf2 signaling pathway in vivo. a The mRNA expressions of Nrf2, HO-1, and NQ01 in mice midbrain were determined by real-time RT-PCR . b, c The protein expressions of nuclear Nrf2 and PCNA, cytosol Nrf2 and HSP90, whole cell H0-1 and NQ01 were measured by western blot assay. Results were mean \pm SEM from 6 mice. ${ }^{*} p<0.05$ compared with the control group, $\# p<0.05$ compared with ROT-treated group. 

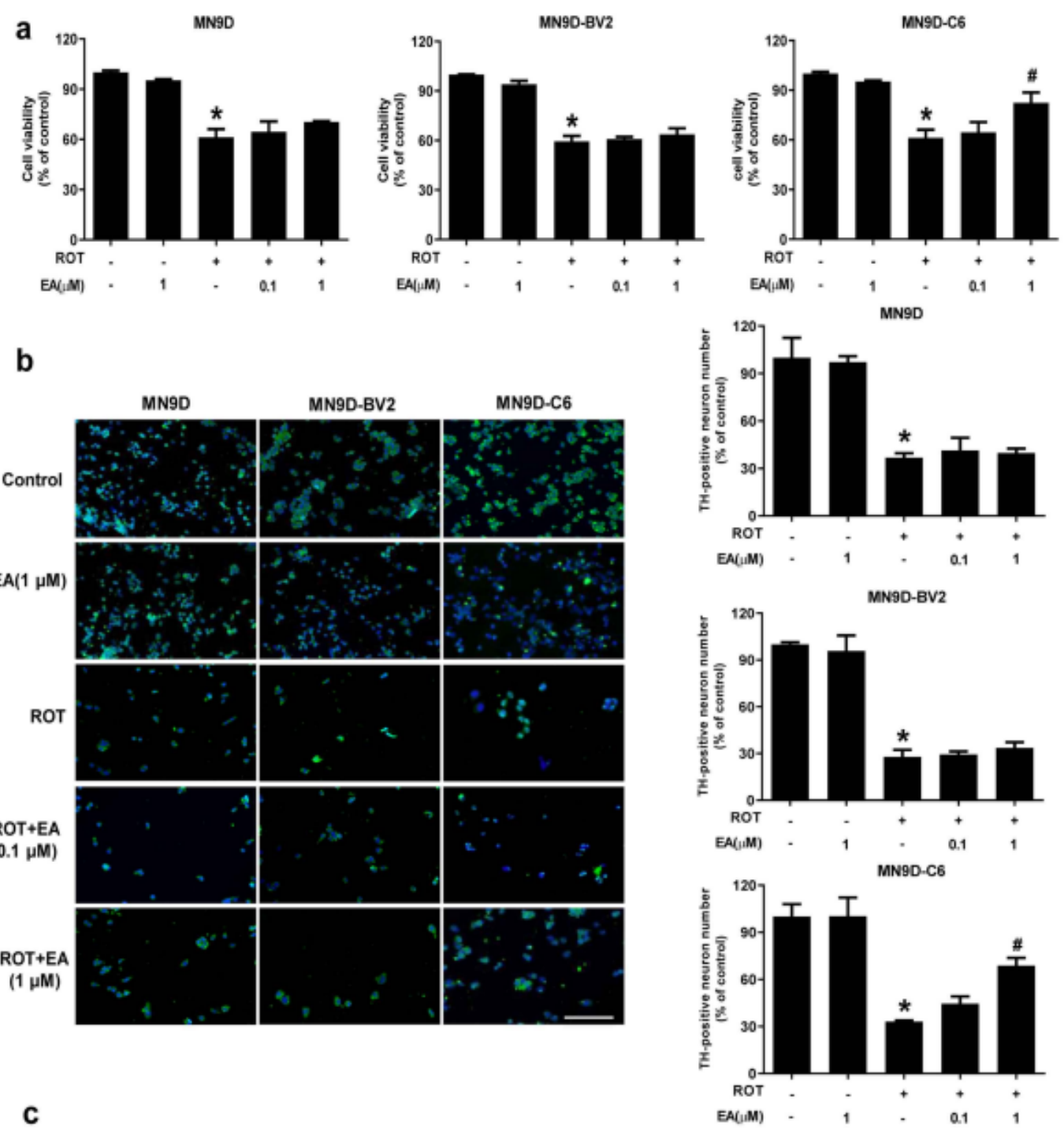

C
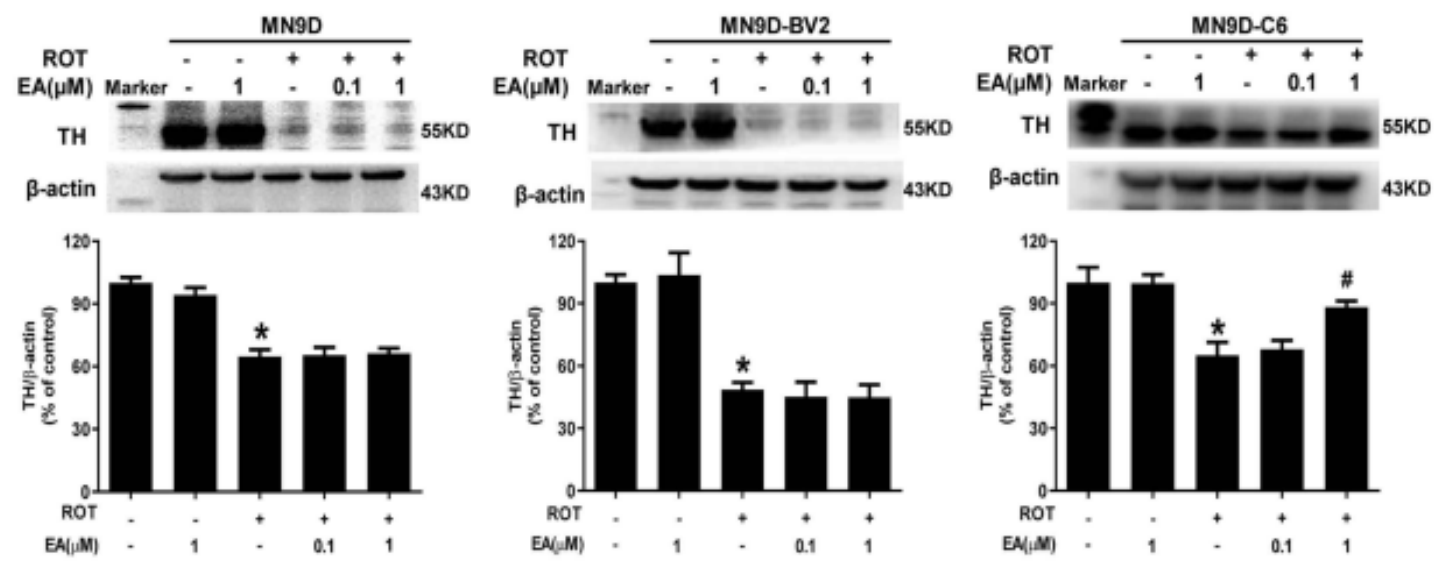

\section{Figure 3}

EA targeted astroglia to protect against ROT-induced DA neuronal damage. MN9D-enriched cultures, MN9D-BV2 and MN9D-C6 co-cultures were cultured for $24 \mathrm{~h}$, respectively. EA was pretreated for 30 min and then incubated with ROT for another $24 \mathrm{~h}$. a The neurotoxicity of ROT and EA on cell viability in MN9D, MN9D-BV2 and MN9D-C6 cultures was determined by MTT assay. b DA neuron number in these 3 cultures was counted after TH-positive neurons immunofluorescence staining. Scale bar $=100 \mu \mathrm{m}$. c TH 
protein expression in 3 cell systems was detected by western blot assay. Results were mean \pm SEM from three independent experiments. ${ }^{*} p<0.05$ compared with the control cultures, $\# p<0.05$ compared with ROTtreated cultures.

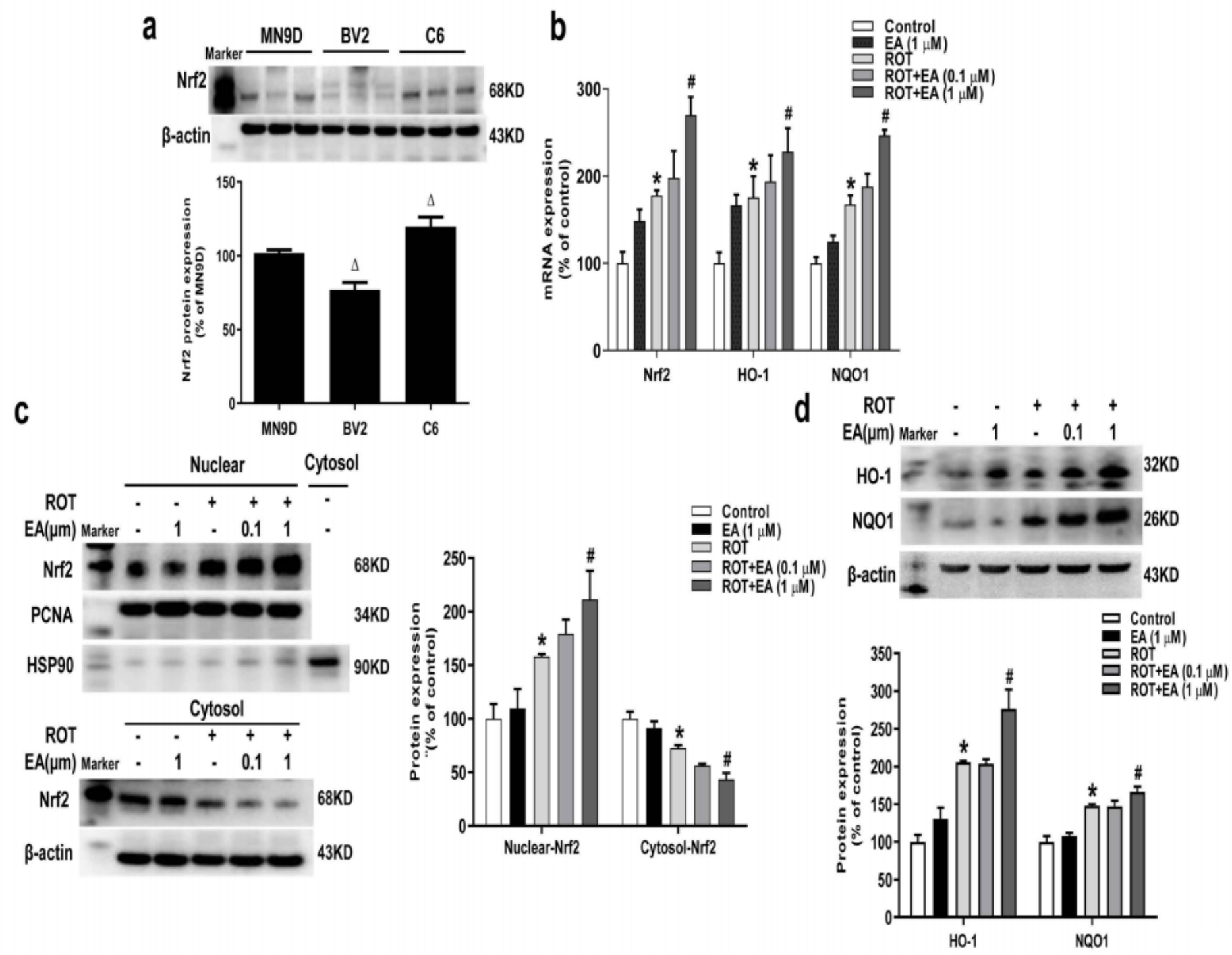

\section{Figure 4}

EA activated Nrf2 signaling pathway in vitro. a Protein expression of Nrf2 in MN9D, BV2 and C6 cultures was detected by western blot assay. $\mathrm{C} 6$ cells were pretreated with $\mathrm{EA}(0.1$ and $1 \mu \mathrm{M})$ for 30 min and then stimulated by ROT $(0.1 \mu \mathrm{M})$ for $24 \mathrm{~h}$. b The mRNA expressions of Nrf2, HO-1 and NQO1 were determined by real-time RT-PCR. c, d The protein expressions of nuclear Nrf2 and PCNA, cytosol Nrf2 and HSP90, whole cell HO-1 and NQO1 were measured by western blot assay. Results were mean \pm SEM from three independent experiments performed in triplicate. ${ }^{*} p<0.05$ compared with the control cultures, $\# p<0.05$ compared with ROT-treated cultures, $\Delta p<0.05$ compared with MN9D cell cultures. 
a
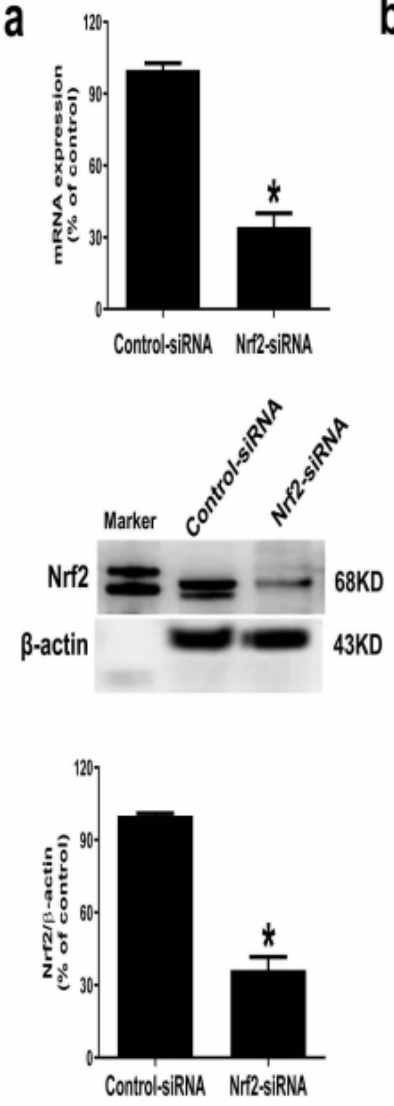

b

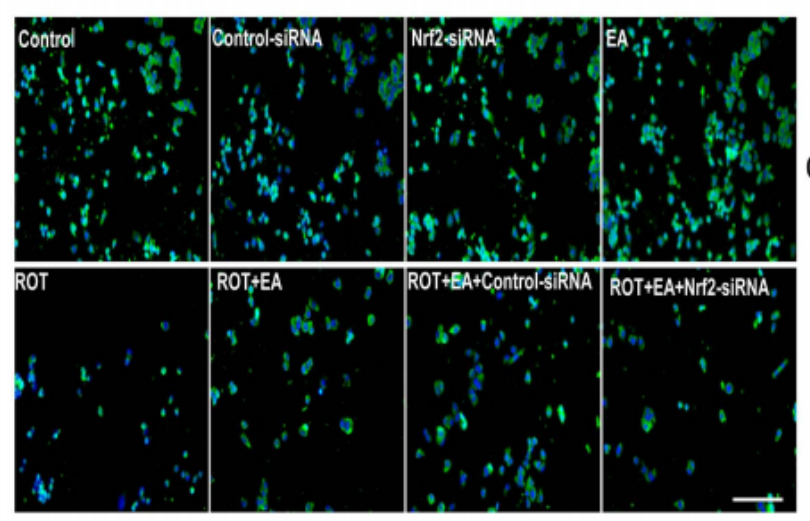

MN9D-C6

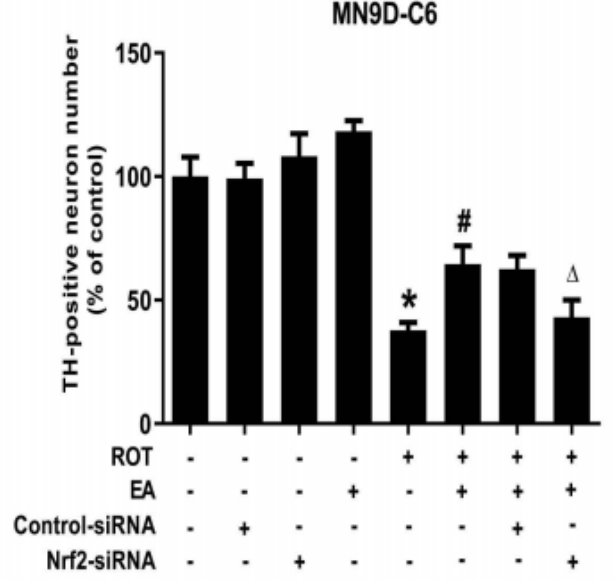

C
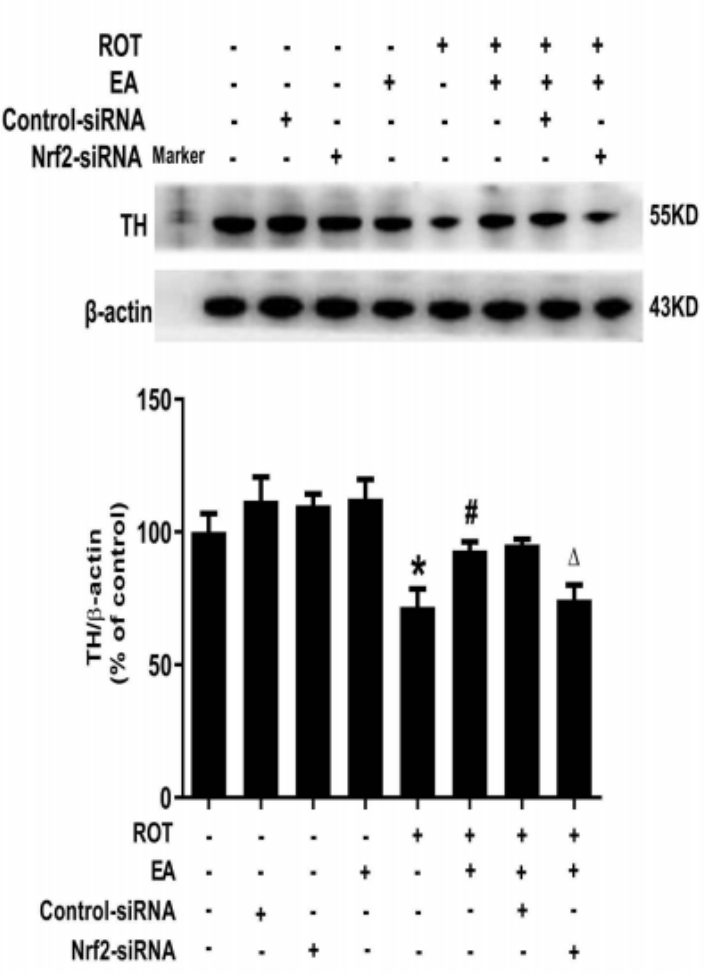

\section{Figure 5}

Role of Nrf2 in EA-mediated DA neuroprotection. C6 cells were treated with Nrf2 siRNA ( $50 \mathrm{nmol} / \mathrm{L})$. After $6 \mathrm{~h}$ of transfection, the transfection solution was removed and cells were rinsed with PBS. a The silence efficiency was validated by real-time RT-PCR and western blotting. Then, C6 cells conditionally silencing Nrf2 were seeded in transwells upper chamber followed by MN9D cells seeded in the lower chamber to establish MD9D-C6 co-cultures and then treated with EA for 30 min and ROT for another 24 h. b, c DA neuronal damage was determined by $\mathrm{TH}$-positive neuronal number counting and $\mathrm{TH}$ protein expression detection. Scale bar $=100 \mu \mathrm{m}$. Results were mean \pm SEM from three independent experiments performed in triplicate. ${ }^{*} \mathrm{p}<0.05$ compared with the control cultures, $\# \mathrm{p}<0.05$ compared with ROT-treated cultures, $\triangle \mathrm{p}<0.05$ compared with $\mathrm{ROT}+$ EA cultures. 
a

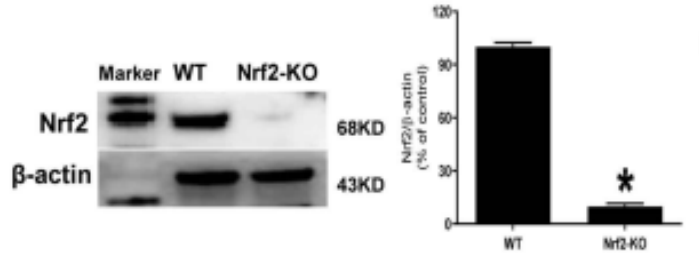

b

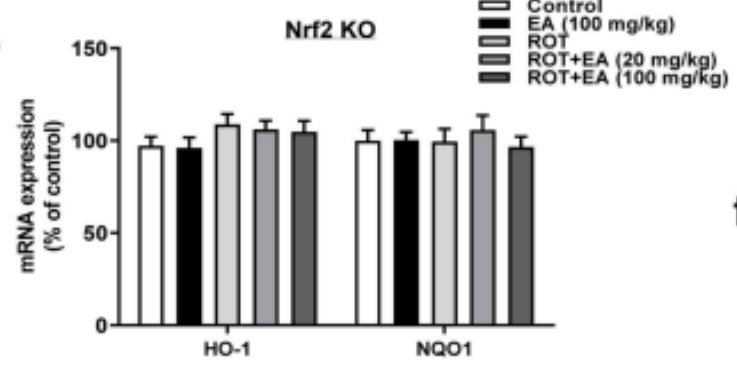

C
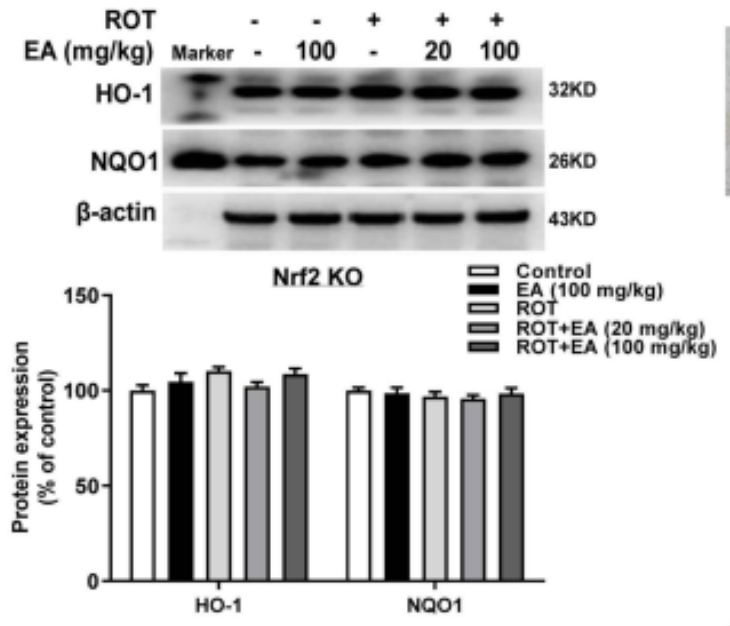

d
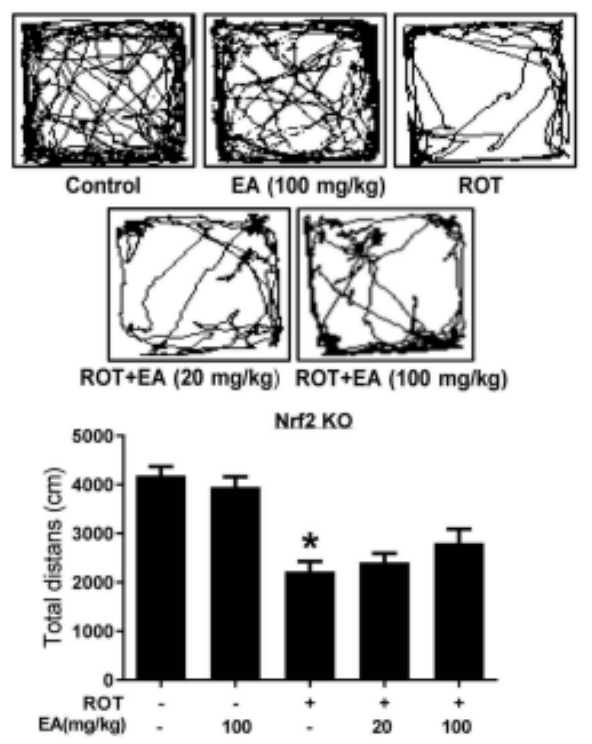

e

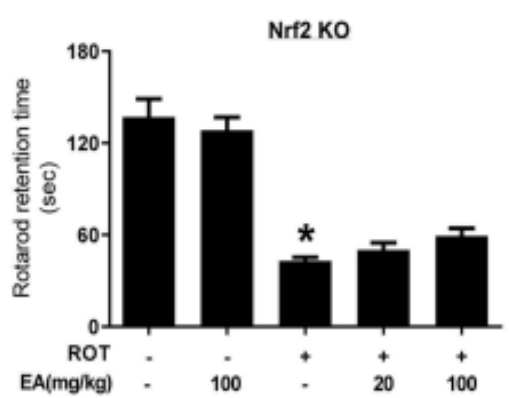

f
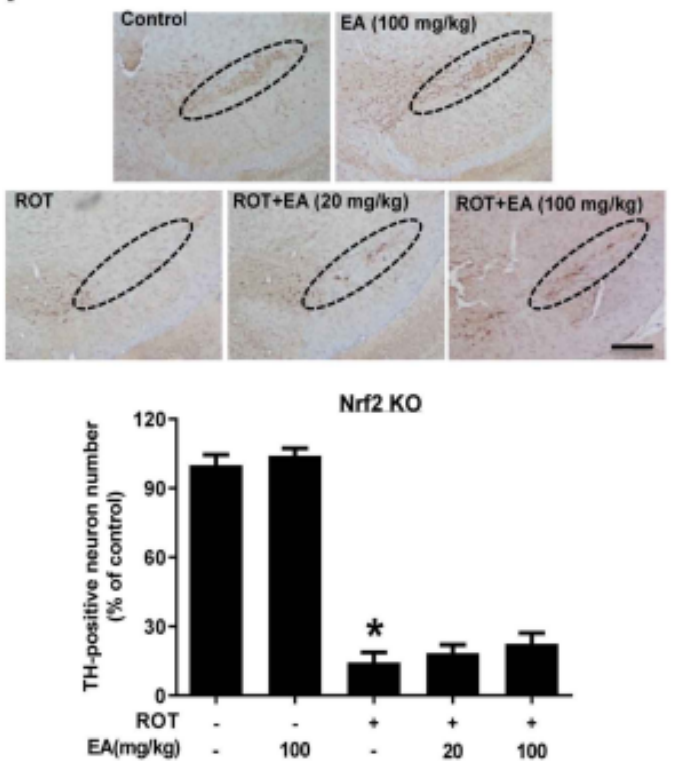

g
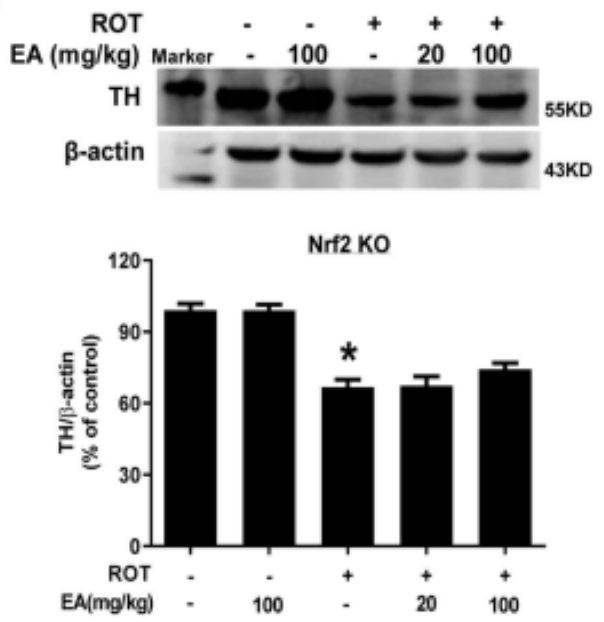

\section{Figure 6}

EA-elicited neuroprotection was abolished in Nrf2 knockout mice. Nrf2 knockout mice received subcutaneous injection of ROT ( $1 \mathrm{mg} / \mathrm{kg}$ ) six times a week for consecutive five weeks followed by intragastric administration of EA for another five weeks. a Western blotting was used to test knockout efficiency in Nrf2 knockout mice. b, c The mRNA and protein expressions of HO-1 and NQ01 in mice midbrain were detected via real-time RT-PCR and western blot assays. $d$, e Mice behavior changes were 
assessed by open field and rotarod tests. $f$ Brain sections were immunostained and the number of THpositive neurons in mice SN was counted. The "ellipse" presented the area of SN. Scale bar $=200 \mu \mathrm{m} . \mathrm{g}$ TH protein expression in SN was measured by western blot assay. Results were mean \pm SEM from 6 mice. ${ }^{*} p<0.05$ compared with the control group. 